\title{
Nicht Entzündung, sondern Schmerz befeuert krankhafte Müdigkeit
}

35-80 \% der Rheumapatienten leiden unter Fatigue. Allein diese Information kann manche motivieren, dem Teufelskreis von Hilflosigkeit und Inaktivität zu entfliehen. Zudem bedarf es psychosozialer Unterstützung und einer guten Schmerzkontrolle.

\section{Fatigue ist häufig bei Rheuma,} die Auswirkungen auf das physische und psychosoziale Wohlbefinden sind mitunter enorm. Im Detail verstanden ist Fatigue nicht. Doch sie wird vor allem durch Schmerz unterhalten. Da Ärzte eher auf Entzündung und andere körperliche Symptome fokussieren, fühlen sich die Patienten oft allein gelassen. Besser, man fragt sie gezielt nach Fatigue und danach, wie diese ihren Alltag beeinflusst.

Zu wissen, dass viele Mitpatienten das gleiche Problem haben, helfe oft, „auch wenn wir keine schnelle Lösung für sie haben", so Prof. Jette Primdahl, registrierte Krankenschwester, Professorin für Rheumatologie, Universitätsklinikum Sønderberg, Dänemark, beim EULAR-Kongress.

Fibromyalgiepatienten klagen mit $82 \%$ am häufigsten über Fatigue. Unter den entzündlich-rheumatischen Erkrankung ist sie am häufigsten bei Psoriasisarthritis mit $57 \%$, gefolgt von $52 \%$ bei Systemischem Lupus erythematodes. Bei rheumatoider Arthritis (RA) und Spondyloarthritis sind es weniger: $41 \%$ und $45 \%$.

\section{Keine normale Müdigkeit}

Als multidimensionales Symptom hat Fatigue körperliche, kognitive und emotionale, sowie persönliche, Verhaltens- und soziale Aspekte. „Fatigue ist anders als normale Müdigkeit oder Schläfrigkeit", so Primdahl. Sie wird intensiver empfunden, als Erschöpfung auch ohne Energieeinsatz, die sich durch Ruhe nicht bessert. Sie kann dauerhaft vorhanden sein oder fluktuieren.

Anstatt, wie lange angenommen, mit der Inflammation korreliert sie am stärksten mit der Schmerzintensität. Eine signifikante Assoziation zu CRP und BSG besteht nicht, dafür zu schlechtem Schlaf, Depression, Sorgen, Ängsten und Krankheitswahrnehmung. Komorbiditäten, Anämie und Medikamentennebenwirkungen können zu Fatigue beitragen. Die Patienten, überwiegend Frauen, fühlen sich hilflos, ohne Kontrolle, ihrem Alltag nicht gewachsen.

Als facettenreiches subjektives Symptom lässt sich Fatigue schwer messen. Für RA-Patienten empfahl Primdahl den Bristol Rheumatoid Arthritis Fatigue Multi-Dimensional Questionnaire (BRAF-MDQ) und die Bristol Rheumatoid Arthritis Fatigue
Numerical Rating Scales (BRAF-NRS). Beide sind auf Deutsch validiert und frei im Internet zugänglich [1].

\section{Schmerzkontrolle und körperliche Aktivität}

Therapeutisch ist vor allem etwas gegen die Schmerzen zu tun. Das gelingt indirekt natürlich zum Teil auch durch medikamentöse Kontrolle einer entzündlichen Krankheitsaktivität. Der wichtigste nicht-pharmakologische Ansatz ist regelmäßige körperliche Aktivität. Das sei für die Patienten oft "sehr hart". Doch selbst weniger zu sitzen oder zu liegen habe schon einen Effekt. „Sie müssen kein hochintensives Training haben, sie sollten nur aktiver sein", so Primdahl. „Und dann sollten wir in der Lage sein, psychosoziale Unterstützung anzubieten." Dazu gehören Aufklärung und Motivation, damit Patienten lernen, aktiv zu werden, sich Ziele zu setzen, ihre Kräfte einzuteilen und sich bei alledem helfen zu lassen, gegebenenfalls auch im Rahmen einer Verhaltenstherapie.

\section{Literatur \\ 1. Bristol Rheumatoid Arthritis Fatigue Scales: https://bit.ly/3dXsDe7}

\section{Das Wichtigste in Kürze}

Welche Bedeutung hat Fatigue bei Rheumapatienten?

Die Prävalenz der Fatigue bei rheumatischen Erkrankungen ist hoch. $35-80 \%$ der Patienten sind betroffen.

Bedeutung: Fatigue ist ein multidimensionales Symptom mit vielen Facetten und mitunter erheblichen negativen biopsychosozialen Folgen. Am stärksten ist Fatigue mit den wahrgenommenen Schmerzen assoziiert.

Empfehlung: Es ist wichtig, dass Ärzte die Fatigue ihrer Patienten thematisieren, um sie letztlich in ihrer Selbstwirksamkeit zu stärken und zu einem aktiveren Leben zu motivieren. Letzteres fällt vielen Patienten schwer.
Hinweis des Verlags. Der Verlag bleibt in Hinblick auf geografische Zuordnungen und Gebietsbezeichnungen in veröffentlichten Karten und Institutsadressen neutral.

rheuma plus $2021 \cdot 20: 206$ https://doi.org/10.1007/s12688-02100452-9

(c) Springer-Verlag GmbH Austria, ein Teil von Springer Nature 2021 